\title{
ON THE EXISTENCE OF CLASSICAL SOLUTIONS FOR DIFFERENTIAL-FUNCTIONAL IBVP
}

\author{
KRZYSZTOF A. TOPOLSKI
}

\begin{abstract}
We consider the initial-boundary value problem for second order differential-functional equations of parabolic type. Functional dependence in the equation is of the Hale type. By using Leray-Schauder theorem we prove the existence of classical solutions. Our formulation and results cover a large class of parabolic problems both with a deviated argument and integrodifferential equations.
\end{abstract}

\section{Introduction.}

Parabolic differential - functional equations have applications in different branches of knowledge. Equations with a retarded argument and differentialintegral equations play important role in biology and physic. Differential functional equations are also interesting from the purely mathematical point of view as they cause much more problems then those without functional dependence. In this paper we consider a very general model of functional dependence (see (3)) containing two types mentioned.

Let $\Omega \subseteq \mathbb{R}^{n}$ be any open bounded domain and $T>0, a_{0}, \tau \in \mathbb{R}_{+}=$ $[0, \infty)$ given constants. Define

$$
\begin{gathered}
\Omega_{\tau}=\left\{x \in \mathbb{R}^{n}: \operatorname{dist}(x, \Omega) \leq \tau\right\}, \quad \partial_{0} \Omega=\Omega_{\tau} \backslash \Omega, \quad \Theta=(0, T) \times \Omega, \\
\Theta_{0}=\left[-a_{0}, 0\right] \times \Omega_{\tau}, \quad \partial_{0} \Theta=(0, T) \times \partial_{0} \Omega, \quad \Gamma=\Theta_{0} \cup \partial_{0} \Theta, \quad E=\Gamma \cup \Theta .
\end{gathered}
$$

Let $D=\left[-a_{0}, 0\right] \times B(\tau)$, where $B(\tau)=\left\{x \in \mathbb{R}^{n}:|x| \leq \tau\right\}$ and $|\cdot|$ is the norm in $\mathbb{R}^{n}$. For every $z: E \rightarrow \mathbb{R}$ and $(t, x) \in \Theta$ we define a function $z_{(t, x)}: D \rightarrow \mathbb{R}$ by $z_{(t, x)}(s, y)=z(t+s, x+y)$ for $(s, y) \in D$. We will call this

1991 Mathematics Subject Classification. 35D05, 35K60, 35R10.

Key words and phrases. Parabolic equation, differential-functional equation, deviated argument.

Supported by KBN Grant 2 PO3A, 01811.

Received: July 25, 1998. 
restriction operator $z \rightarrow z_{(t, x)}$ Hale's operator and functional dependence in the equation "the Hale type" (see [8] and [9]).

Let $a_{i, j}, \quad b_{i} \in C(\bar{\Theta}, \mathbb{R})$ and $\mathcal{L}$ be a second order differential operator defined by

$$
\mathcal{L} z(t, x)=D_{t} z(t, x)-\sum_{i, j=1}^{n} a_{i, j}(t, x) D_{x_{i} x_{j}}^{2} z(t, x)-\sum_{i=1}^{n} b_{i}(t, x) D_{x_{i}} z(t, x) .
$$

In the following we will assume than $\mathcal{L}$ is strictly uniformly parabolic in $\Theta$ i.e. there exists some positive constant $k$ such that

$$
k^{-1}|\xi|^{2} \leq \sum_{i, j=1}^{n} a_{i, j}(t, x) \xi_{i} \xi_{j} \leq k|\xi|^{2}
$$

for all $\xi=\left(\xi_{1}, \ldots, \xi_{n}\right) \in \mathbb{R}^{n}$ and $(t, x) \in \Theta$.

Suppose that $f: \Theta \times \mathbb{R} \times C(D, \mathbb{R}) \times \mathbb{R}^{n} \rightarrow \mathbb{R}$ of the variables $(t, x, u, w, p)$ and $\Psi: \Gamma \rightarrow \mathbb{R}$ are given function. We will consider the initial-boundary value problem (IBVP) for second order differential-functional equation in the following form,

$$
\begin{array}{rlrl}
\mathcal{L} u(t, x) & =f\left(t, x, u(t, x), u_{(t, x)}, D_{x} u(t, x)\right) & & \text { in } \Theta, \\
u(t, x) & =\Psi(t, x) & \text { in } \Gamma .
\end{array}
$$

Although the formulation of the above problem seems to be rather abstract, it contains as a particular case equations with a deviated argument and a few kinds of differential-integral equations. This can be derived from $(3),(4)$ by specializing the function $f$.

Indeed, consider two examples,

Example 1.1. Let $g: \Theta \times \mathbb{R} \times \mathbb{R} \times \mathbb{R}^{n} \rightarrow \mathbb{R}$ and $\mu: \Theta \rightarrow \mathbb{R}, \nu: \Theta \rightarrow \mathbb{R}^{n}$ are given function such that

$$
t-a_{0} \leq \mu(t, x) \leq t \quad|\nu(t, x)-x| \leq \tau \quad \text { for }(t, x) \in \Theta .
$$

Consider IBVP problem

(6) $\mathcal{L} u(t, x)=g\left(t, x, u(t, x), u(\mu(t, x), \nu(t, x)), D_{x} u(t, x)\right) \quad$ in $\quad \Theta$,

(7) $u(t, x)=\Psi(t, x)$ in $\Gamma$.

It is easy to verify that putting

$$
f(t, x, u, w, p)=g(t, x, u, w(\mu(t, x)-t, \sigma(t, x)-x), p)
$$

for $(t, x, u, w, p) \in \Theta \times \mathbb{R} \times C(D, \mathbb{R}) \times \mathbb{R}^{n}$ we can obtain problem (3),(4) (put $u_{(t, x)}$ in place of $w$ ).

In Section 3 we present the theorem on the existence of classical solution for $(6),(7)$.

Problem in the form (3), (4) can be obtained also by transformation of differential-integral equation. 
Example 1.2. Let $D_{(t, x)}=\left\{\left(t+t^{\prime}, x+x^{\prime}\right):\left(t^{\prime}, x^{\prime}\right) \in D\right\}$ and $h: \Theta \times \mathbb{R} \times$ $\mathbb{R} \times \mathbb{R}^{n} \rightarrow \mathbb{R}, K: \Theta \times E \times \mathbb{R} \rightarrow \mathbb{R}$ are given function .

Consider problem,

$(8) \mathcal{L} u(t, x)=h\left(t, x, u(t, x), \int_{D_{(t, x)}} K(t, x, s, y, u(s, y)) d s d y, D_{x} u(t, x)\right)$ in $\Theta$

(9) $u(t, x)=\Psi(t, x)$

in $\Gamma$.

Define $f: \Theta \times \mathbb{R} \times C(D, \mathbb{R}) \times \mathbb{R}^{n} \rightarrow R$ by

$$
f(t, x, u, w, p)=h\left(t, x, u, \int_{D} K(t, x, t+s, x+y, w(s, y)) d s d y, p\right)
$$

By the above formula it is evident that (8),(9) can be treated as a particular case of (3), (4).

Of course, using similar argument, we can also combine these two kinds of functional dependens and treat them using one model.

The natural question that arise here is how to formulate asumption on $f$ in rather abstract case $(3),(4)$ in order to obtain interesting theorems for $(6),(7)$ and $(8),(9)$. The purpose of our paper is to prove existence theorem for $(3),(4)$ general enough to cover presented examples. We will concentrate on the first example as it require more careful treatment (see explanation after Assumption 2.1).

As we mentioned at the begining there are numerous applications of parabolic differential-functional equations. In Volterra-Lotka model with two competing species the functions describing the growth rate are solutions of parabolic equations with time delays. When the Volterra-Lotka competition model involves $m$ competing species where the reaction rate depends on the functional values of the species, the governing equations for the population densitties are a weakly coupled parabolic differential-integral system (see $[12])$.

The linearized theory of rigid conductors of heat, composed of materials with memory, leads to parabolic differential-integral equation (see [2]). The paper [7] deals with the delay reaction-diffusion equation. Global solutions and asymptotic behaviour are investigated. This equation describes the evolution of a single diffusing animal species. The growth rate reaction to population density changes includes the delay term which involves the entire past history. Reaction-diffusion integral equations are studied, with special regard to explosive-type solutions, in [16].

The paper [12] deals with weakly coupled parabolic systems with time delays. Differential-integral problems are considered also. It is shown by using upper and lower solutions and by monotone iterative techniques that the coresponding sequences of approximate solutions are convergent monotonically to a unique solutions of the original problem. Given functions in nonlinear parts of systems satisfy the Lipschiz condition and fulfil the mixed quasimonotone property. 
A few monotone iterative methods have been applied in $[5,6]$ to studying existence problems for nonlinear parabolic differential -functional equations. Only a small class of differential-integral equations satisfies all the assumptions of existence theorem given in $[5,6]$. Equations with a deviated argument are not covered by this theory.

The main difficulty in appllying monotone iterative methods is a construction of a lower and an upper functions. General methods of finding these functions are little described in literature.

In the paper we do not need assumptions on lover and upper functions. We also consider larger class of problems. The function $f$ depends also on $D_{x} u$ in our model.

It must be said also that, although we focus on one equation, our result can be extended on weakly coupled systems in an obvious way. We do not assume quasimonotone conditions in this case. The function $f$ depends also on $D_{x} u$

For problems without functional dependence on $u$ this subject was investigated in [10] and [1]. It is worth to be mentioned, that other type of functional dependence, for first equations, is treated in $[3,4]$.

The classical works on the uniqueness for parabolic differential-functional equations are $[13,14]$. This problem for equation in the form (3) is studied in [15] were the author consider solutions in generalized sense.

We will investigate classical solutions of $(3),(4)$. We will use the symbol $C L S(f, \Psi, \mathcal{L})$ for the set of all classical solution of $(3),(4)$.

A function $\omega: \mathbb{R}_{+} \mapsto \mathbb{R}_{+}$is called modulus if $\omega$ is nondecreasing and $\omega\left(0^{+}\right)=0$. Let $C(D, \mathbb{R}, R)=\left\{w \in C(D, \mathbb{R}):\|w\|_{D} \leq R\right\}$ where $\|\cdot\|_{A}$ is the supremum norm in the space $C(D, \mathbb{R})$

We write $G_{t}=\left\{(s, x) \in G:-a_{0} \leq s \leq t\right\}$ for any $G \subseteq \mathbb{R}^{n+1}$.

Let $M \geq 0$. We will write $\sigma \in O_{M}$ if $\sigma:[0, T] \times \mathbb{R}_{+} \mapsto \mathbb{R}_{+}$is continuous and nondecreasing, with respect to both variables, function such that, the right-hand maximum solution of the problem

$$
z^{\prime}(t)=\sigma(t, z(t)), \quad z(0)=M .
$$

exists in $[0, T]$. We will denote this solution by $\mu_{\sigma}(\cdot, M)$.

Definition 1.1. Let $M \geq 0, \sigma \in O_{M}$. We will write $f \in X_{\sigma, M}$ if

(i) for every $(t, x, r, w) \in \Theta \times \mathbb{R} \times C(D, \mathbb{R})$

$$
f(t, x, r, w, 0) \operatorname{sgn}(r) \leq \sigma\left(t, \max \left(|r|,\|w\|_{D}\right)\right) ;
$$

(ii) for every $R>0$ there exists modulus $\omega_{R}$ such that $|f(t, x, r, w, p)-f(t, x, r, w, 0)| \leq \omega_{R}(|p|) \quad$ in $\quad \Theta \times[-R, R] \times C(D, \mathbb{R}, R) \times \mathbb{R}^{n}$.

Let

$$
\tilde{R}=\tilde{R}(\sigma, M)=\mu_{\sigma}(T, M) .
$$

Proposition 1.1. Suppose that $f \in X_{\sigma, M},\|\Psi\|_{\Gamma} \leq M$ and $u \in C L S(f, \Psi, \mathcal{L})$. Then

$$
\|u\|_{E_{t}} \leq \mu_{\sigma}(t, M) \leq \tilde{R}=\tilde{R}(\sigma, M) \quad \text { for } \quad t \in[0, T]
$$


This Proposition is proved in [15] ( Theorem 2 ).

Definition 1.2. Let $(X,\|\cdot\|)$ be a real metric space, and $R \geq 0$ any constant. We define $I_{R}: X \mapsto X$ by

$$
I_{R}(x)= \begin{cases}x, & \text { if }\|x\| \leq R ; \\ \frac{x}{\|x\|} R & \text { if }\|x\|>R .\end{cases}
$$

It is evident that

$$
\left\|I_{R}(x)\right\|=\min (\|x\|, R), \quad\left\|I_{R}(x)-I_{R}(y)\right\| \leq\|x-y\| \quad \text { in } \quad X .
$$

Let now $I_{R}^{*}: \mathbb{R} \rightarrow \mathbb{R}$ and $I_{R}: C(D, \mathbb{R}) \mapsto C(D, \mathbb{R})$ be defined by (13). For any function $f: \Theta \times \mathbb{R} \times C(D, \mathbb{R}) \times \mathbb{R}^{n} \rightarrow \mathbb{R}$ we define $f_{R}$ : $\Theta \times \mathbb{R} \times C(D, \mathbb{R}) \times \mathbb{R}^{n} \rightarrow \mathbb{R}$ by,

$$
f_{R}(t, x, u, w, p)=f\left(t, x, I_{R}^{*}(u), I_{R}(w), p\right) .
$$

By the definition of $f_{R}$ and Proposition 1.1 we have at once,

Remark 1.1. Let $\|\Psi\|_{\Gamma} \leq M, \sigma \in 0_{M}, \tilde{R}=\tilde{R}(\sigma, M)$. If $f \in X_{\sigma, M}$ then,

(i) $f_{R} \in X_{\sigma, M}$;

(ii) $C L S(f, \Psi, \mathcal{L})=C L S\left(f_{R}, \Psi, \mathcal{L}\right)$.

\section{Existence OF SOLUtion OF IBVP.}

Let $C^{1,2}(\bar{\Theta}, \mathbb{R})$ denotes the space of all function $u \in C(\bar{\Theta}, \mathbb{R})$ such that $D_{t} u, D_{x} u, D_{x}^{2} u$ exist and are continuous in $\bar{\Theta}$. Write $C_{*}^{1,2}(E, \mathbb{R})=C^{1,2}(\bar{\Theta}, \mathbb{R}) \cap$ $C(\bar{E}, \mathbb{R})$. In the following we will always assume that there exists $\tilde{\Psi} \in$ $C_{*}^{1,2}(E, \mathbb{R})$ such that $\tilde{\Psi}_{\mid \Gamma}=\Psi$.

Definition 2.1. We will say that $\operatorname{IBVP}(3),(4)$ satisfies the compatibility condition if

$$
\begin{gathered}
D_{t} \Psi(0, x)-\sum_{i, j=1}^{n} a_{i j}(0, x) D_{x_{i} x_{j}} \Psi(0, x)-\sum_{i}^{n} b_{i}(0, x) D_{x_{i}} \Psi(0, x) \\
=f\left(0, x, \Psi(0, x), \Psi_{(0, x)}, D_{x} \Psi(0, x)\right)
\end{gathered}
$$

for $x \in \partial \Omega$.

Let $A \subseteq \mathbb{R}^{1+n}$ any bounded domain and $\alpha \in(0,1), \quad l=\alpha, 1+\alpha, 2+\alpha$. We will denote by $C^{l / 2, l}(A, \mathbb{R})$ the space of all function $u: A \rightarrow \mathbb{R}$ of the variable $(t, x)$ such that $D_{x}^{r} u$ (for $\left.r=0, \ldots[l]\right)$ and $D_{t}^{k} u$ (for $k=0,1 \ldots[l / 2]$ ) exist and are continuous in $A, D_{x}^{[l]} u$ satisfies Hölder condition with exponent $l-[l]$ with respect to $x$ and $D_{t}^{[l / 2]} u$ satisfies Hölder condition with exponent $l / 2-[l / 2]$ with respect to $t$. We will use the symbol $\|\cdot\|_{l}^{A}\left(\right.$ or $\left.\|\cdot\|_{l}\right)$ to denote the norm in $C^{l / 2, l}(A, \mathbb{R})$. For the properties of the space $C^{l / 2, l}(A, \mathbb{R})$ we refer the reader to $[10]$. It is well known that $\left(C^{l / 2, l}(A, \mathbb{R}),\|\cdot\|_{l}\right)$ is a Banach space. 
Let $\bar{\beta}, \beta \in(0,1]$. We will also consider the space $C^{\bar{\beta}, \beta}(A, \mathbb{R})$ with the norm

$$
\|u\|_{\bar{\beta}, \beta}^{A}=\|u\|_{A}+H_{t, A}^{\bar{\beta}}(u)+H_{x, A}^{\beta}(u)
$$

where

$$
\begin{gathered}
H_{t, A}^{\bar{\beta}}(u)=\sup \left\{\frac{|u(t, x)-u(\bar{t}, x)|}{|t-\bar{t}|^{\bar{\beta}}}:(t, x),(\bar{t}, x) \in A, t \neq \bar{t}\right\}, \\
H_{x, A}^{\beta}(u)=\sup \left\{\frac{|u(t, x)-u(t, \bar{x})|}{|x-\bar{x}|^{\beta}}:(t, x),(t, \bar{x}) \in A, x \neq \bar{x}\right\},
\end{gathered}
$$

Of course this extends the definition of $C^{\alpha / 2, \alpha}(A, \mathbb{R})$ ( notice that $H_{x, A}^{1}(u)$ denotes the Lipschitz constant in $x$ for $u$ and $\bar{\beta}$ may not be equal to $\beta / 2$ ). Let $C^{\bar{\beta}, \beta}(A, \mathbb{R}, q)=\left\{w \in C^{\bar{\beta}, \beta}(A, \mathbb{R}):\|w\|_{\bar{\beta}, \beta}^{A} \leq q\right\}$

Write

$$
\begin{gathered}
C_{\bar{\beta}, \beta}^{(1+\alpha) / 2,1+\alpha}(E, \mathbb{R})=C^{\bar{\beta}, \beta}(\bar{E}, \mathbb{R}) \cap C^{(1+\alpha) / 2,1+\alpha}(\bar{\Theta}, \mathbb{R}) \\
C_{\bar{\beta}, \beta}^{1+\alpha / 2,2+\alpha}(E, \mathbb{R})=C^{\bar{\beta}, \beta}(\bar{E}, \mathbb{R}) \cap C^{1+\alpha / 2,2+\alpha}(\bar{\Theta}, \mathbb{R})
\end{gathered}
$$

with norms

$$
\|u\|_{1+\alpha}^{\bar{\beta}, \beta}=\max \left(\|u\|_{\bar{\beta}, \beta}^{E},\|u\|_{1+\alpha}^{\Theta}\right), \quad\|u\|_{2+\alpha}^{\bar{\beta}, \beta}=\max \left(\|u\|_{\bar{\beta}, \beta}^{E},\|u\|_{2+\alpha}^{\Theta}\right) .
$$

Let

$$
C_{*}^{0,1}(E, \mathbb{R})=C(\bar{E}, R) \cap C^{0,1}(\bar{\Theta}, \mathbb{R}) \quad \text { and } \quad\|u\|_{0,1}^{*}=\|u\|_{0}^{E}+\left\|D_{x} u\right\|_{0}^{\bar{\Theta}} .
$$

In the following $\alpha \in(0,1), \alpha \leq \gamma \leq 1$ are given constants. Our basic assumption is the following.

Assumption $2.1\left(A_{\bar{\beta}, \beta}\right)$. Let $\|\Psi\|_{\Gamma} \leq M$, and $\frac{\alpha+1}{2} \geq \bar{\beta} \geq \frac{\alpha}{2 \gamma}, 1 \geq \beta \geq \frac{\alpha}{\gamma}$ given constants. Suppose that,

1) there exists $\sigma \in O_{M}$ such that $f \in X_{\sigma, M}$;

2) there exists nondecreasing function $\rho: \mathbb{R}_{+} \rightarrow \mathbb{R}_{+}$such that

$$
|f(t, x, u, w, p)| \leq \rho\left(\max \left(|u|,\|w\|_{D}\right)\left(1+|p|^{2}\right)\right.
$$

in $\Theta \times \mathbb{R} \times C(D, \mathbb{R}) \times \mathbb{R}^{n}$;

3) for every $R, L \geq 0$ there exists a constant $C(R, L) \geq 0$ such that $|f(t, x, u, w, p)-f(t, x, \bar{u}, \bar{w}, \bar{p})| \leq C(R, L)\left(|u-\bar{u}|^{\alpha}+\|w-\bar{w}\|_{D}^{\gamma}+|p-\bar{p}|\right)$ on $\Theta \times[-R, R] \times C(D, \mathbb{R}, R) \times B(L)$;

4) for every $R, q, L \geq 0$ there exists a constant $H(R, q, L) \geq 0$ such that

$$
|f(t, x, u, w, p)-f(\bar{t}, \bar{x}, u, w, p)| \leq H(R, q, L)\left(|t-\bar{t}|^{\alpha / 2}+|x-\bar{x}|^{\alpha}\right)
$$

on $\Theta \times[-R, R] \times C^{\bar{\beta}, \beta}(D, \mathbb{R}, q) \times B(L)$;

5) there exists $\tilde{\Psi} \in C_{\bar{\beta}, \beta}^{1+\alpha / 2,2+\alpha}(E, \mathbb{R})$ such that $\tilde{\Psi}_{\mid \Gamma}=\Psi$. 
Since our assumptions could be not clear enough for some of the readers we should give a little explanation. We introduce 1) to get apriori bound on solutions for problem (3) (4). The second item is a functional form of Nagumo condition (see [10]). It plays important role in obtaining apriori bound on $x$-derivative of the solutions. Notice that the Lipschitz-Hölder condition on $f$ is divided into 3 ) and 4 ). It has its meaning. The fact that we take the space $C^{\bar{\beta}, \beta}(D, \mathbb{R}, q)$ in 4$)$ allows us to apply our results not only to differential-integral equations but to equations with a deviated argument as well (see the last paragraph). It would not be possible if we took in 4) larger space $C(D, \mathbb{R}, q)$. Of course the assumption would be stronger in this case. One of the reason why we introduce space $C^{\bar{\beta}, \beta}(A, \mathbb{R})$ is that we want to obtain possibly the most general result. Assumming more about $\Psi$ we can assume less about $f$ and vice versa.

Remark 2.1. Suppose that $f$ satisfies Assumption 2.1. Let $\sigma_{0}=$ $\|\sigma(\cdot, \tilde{R})\|_{[0, T]}, \rho_{0}=\rho(\tilde{R})$. It is easy to check that $f_{R}$ defined by (15) satisfies Assumption 2.1 with $\sigma_{0}, \rho_{0}$ in place of $\sigma, \rho$ and with $C(R, L), H(R, q, L)$ independent of $R$.

In view of this remark and Remark 1.1 (ii) without loss of generality we can assume that $C(R, L)=C_{L}, H(R, q, L)=H_{q, L}$.

Define

$$
f_{\tilde{\Psi}}(t, x, u, w, p)=f\left(t, x, u+\tilde{\Psi}(t, x), w+\tilde{\Psi}_{(t, x)}, p+D_{x} \tilde{\Psi}(t, x)\right)-\mathcal{L} \tilde{\Psi}(t, x)
$$

for $\tilde{\Psi} \in C_{*}^{1,2}(E, \mathbb{R})$.

Remark 2.2. If $(f, \Psi)$ satisfy Assumption 2.1 and $a_{i j}, b_{i} \in C^{\alpha / 2, \alpha}(\bar{\Theta}, \mathbb{R})$, then

(i) $\left(f_{\tilde{\Psi}}, 0\right)$ satisfy Assumption 2.1;

(ii) $u \in C L S(f, \Psi, \mathcal{L})$ if and only if $u-\tilde{\Psi} \in C L S\left(f_{\tilde{\Psi}}, 0, \mathcal{L}\right)$.

This, easy to verify, remark allows us to simplify our problem.

We define the Nemytskii operator for problem (3),(4). Put

$$
F(u)(t, x)=f\left(t, x, u(t, x), u_{(t, x)}, D_{x} u(t, x)\right), \quad(t, x) \in \Theta
$$

where $u \in C_{*}^{0,1}(E, \mathbb{R})$.

The following properties of the Nemytskii operator are very useful in the proof of the existence.

Lemma 2.1. Suppose that $f$ satisfies Assumption 2.1 and let $F$ be the Nemytskii operator for (3),(4). Then

(i) $F: C_{*}^{0,1}(E, \mathbb{R}) \rightarrow C(\bar{\Theta}, \mathbb{R})$ is continuous and bounded.

(ii) $F\left(C_{\bar{\beta}, \beta}^{(1+\alpha) / 2,1+\alpha}(E, \mathbb{R})\right) \subseteq C^{\alpha / 2, \alpha}(\bar{\Theta}, \mathbb{R})$. 
Proof. (i) Let $\|u-\bar{u}\|_{0,1}^{*} \leq 1$, and $R=\|\bar{u}\|_{0,1}^{*}+1$. Then

$$
\begin{aligned}
& |F(u)(t, x)-F(\bar{u})(t, x)| \\
& =\left|f\left(t, x, u(t, x), u_{(t, x)}, D_{x} u(t, x)\right)-f\left(t, x, \bar{u}(t, x), \bar{u}_{(t, x)}, D_{x} \bar{u}(t, x)\right)\right| \\
& \leq C_{R}\left(|u(t, x)-\bar{u}(t, x)|^{\alpha}+\left\|u_{(t, x)}-\bar{u}_{(t, x)}\right\|_{D}^{\gamma}+\left|D_{x} u(t, x)-D_{x} \bar{u}(t, x)\right|\right) \\
& \leq C_{R}\|u-\bar{u}\|_{0,1}^{*},
\end{aligned}
$$

which shows that $F$ is continuous.

Let $\|u\|_{0,1}^{*} \leq R$. Since

$$
\begin{aligned}
|(F u)(t, x)| \leq & \left|f\left(t, x, u(t, x), u_{(t, x)}, D_{x} u(t, x)\right)-f(t, x, 0,0,0)\right| \\
& +|f(t, x, 0,0,0)| \\
\leq & C_{R}\left(\|u\|_{\Theta}^{\alpha}+\|u\|_{E}^{\gamma}+\left\|D_{x} u\right\|_{\Theta}\right)+\|f(\cdot, \cdot, 0,0,0)\|_{\Theta},
\end{aligned}
$$

we see that $F$ is bounded.

(ii) Let $\left.u \in C_{\bar{\beta}, \beta}^{(1+\alpha) / 2,1+\alpha}(E, \mathbb{R})\right)$. Then $F u$ is Hölder continuous with respect to $x$. Indeed, Put $R=\|u\|_{1+\alpha}^{\bar{\beta}, \beta}$. Of course this implies $\|u\|_{\bar{\beta}, \beta}^{E} \leq R$ and we have by Assumption 2.1

$$
\begin{aligned}
\mid & (F u)(t, x)-(F u)(t, \bar{x}) \mid \\
= & \left|f\left(t, x, u(t, x), u_{(t, x)}, D_{x} u(t, x)\right)-f\left(t, \bar{x}, u(t, \bar{x}), u_{(t, \bar{x})}, D_{x} u(t, \bar{x})\right)\right| \\
\leq & \left|f\left(t, x, u(t, x), u_{(t, x)}, D_{x} u(t, x)\right)-f\left(t, x, u(t, \bar{x}), u_{(t, \bar{x})}, D_{x} u(t, \bar{x})\right)\right| \\
& +\left|f\left(t, x, u(t, \bar{x}), u_{(t, \bar{x})}, D_{x} u(t, \bar{x})\right)-f\left(t, \bar{x}, u(t, \bar{x}), u_{(t, \bar{x})}, D_{x} u(t, \bar{x})\right)\right| \\
\leq & C_{R}\left(|u(t, x)-u(t, \bar{x})|^{\alpha}+\left\|u_{(t, x)}-u_{(t, \bar{x})}\right\|_{D}^{\gamma}+\left|D_{x} u(t, x)-D_{x} u(t, \bar{x})\right|\right) \\
& +H_{R, R}|x-\bar{x}|^{\alpha} \\
\leq & C_{R}\left(\left[\left\|D_{x} u\right\|_{\Theta}\right]^{\alpha}|x-\bar{x}|^{\alpha}+\left[H_{x, E}^{\beta}(u)\right]^{\gamma}|x-\bar{x}|^{\beta \gamma}+H_{x, \Theta}^{\alpha}\left(D_{x} u\right)|x-\bar{x}|^{\alpha}\right) \\
& +H_{R, R}|x-\bar{x}|^{\alpha},
\end{aligned}
$$

where $H_{x, \Theta}^{\alpha}\left(D_{x} u\right)=\sum_{i=1}^{n} H_{x, \Theta}^{\alpha}\left(D_{x_{i}} u\right)$.

Since $\beta \gamma \geq \alpha$ we get desired conclusion.

Similarly, remembering that $\bar{\beta} \geq \frac{\alpha}{2 \gamma}$, we prove the Hölder continuity of $F u$ with respect to $t$

Lemma 2.2. Suppose that $f, \Psi$ satisfy Assumption 2.1 and $\tilde{u} \in C_{*}^{1,2}(E, \mathbb{R})$ is a solution of (3),(4). Then there exist constant $\tilde{L}$ such that

$$
\left\|D_{x} \tilde{u}\right\|_{0}^{\bar{\Theta}} \leq \tilde{L} .
$$

Proof. Without loss of generality we can assume that $\Psi \equiv 0$. Put $f^{*}$ : $\Theta \times \mathbb{R} \times \mathbb{R}^{n} \rightarrow \mathbb{R}, \quad f^{*}(t, x, v, p)=f\left(t, x, v, \tilde{u}_{(t, x)}, p\right)$.

Consider problem

$$
\begin{aligned}
\mathcal{L} v(t, x) & =f^{*}\left(t, x, v(t, x), D_{x} v(t, x)\right) & & (t, x) \in \Theta \\
v(t, x) & =0 & & (t, x) \in \Sigma
\end{aligned}
$$


It is evident that $\tilde{u}_{/ \bar{\Theta}}$ is a solution of $(19),(20)$. It easy to check that $f^{*}$ satisfies hypotheses of Theorem 2.2 [1]. Therefore there exists a constant $\tilde{L}$ depending on $\mathcal{L}, \Psi, \tilde{R}, \rho$ such that (18) holds true.

Now we are ready to state our main result.

Theorem 2.1. Assume that

1) $a_{i j}, b_{i} \in C^{\alpha / 2, \alpha}(\bar{\Theta}, \mathbb{R})$;

2) $\partial \Omega$ belongs to class $C^{2+\alpha}$;

3) $f, \Psi$ satisfy Assumption 2.1 and the problem (3) (4) satisfies the compatibility condition (16).

Then IBVP (3),(4) has a solution $u \in C_{\bar{\beta}, \beta}^{1+\alpha / 2,2+\alpha}(E, \mathbb{R})$.

Proof. In view of Remark 2.2 we may assume that $\Psi \equiv 0$. The compatibility condition (16) takes now form

$$
f(0, x, 0,0,0)=0 \text { for } \mathrm{x} \in \partial \Omega .
$$

Put $\Sigma=(\{0\} \times \Omega) \cup([0, T] \times \partial \Omega)$. Define,

$$
\begin{gathered}
C_{0}^{\alpha / 2, \alpha}(\bar{\Theta}, \mathbb{R})=\left\{g \in C^{\alpha / 2, \alpha}(\bar{\Theta}, \mathbb{R}): g(0, x)=0 \text { for } x \in \partial \Omega\right\} \\
C^{l / 2, l}(\bar{\Theta}, \mathbb{R}, 0)=\left\{g \in C^{l / 2, l}(\bar{\Theta}, \mathbb{R}): g_{\mid \Sigma}=0\right\} \\
C^{l / 2, l}(E, \mathbb{R}, 0)=\left\{g \in C_{\bar{\beta}, \beta}^{l / 2, l}(E, \mathbb{R}): g_{\mid \Gamma}=0\right\}
\end{gathered}
$$

where $l=1+\alpha, 2+\alpha$. It is evident that we can treat $C^{l / 2, l}(\bar{\Theta}, \mathbb{R}, 0)$ and $C^{l / 2, l}(E, \mathbb{R}, 0)$ as one space.

Let us define the operator $V: C_{0}^{\alpha / 2, \alpha}(\bar{\Theta}, \mathbb{R}) \rightarrow C^{1+\alpha / 2,2+\alpha}(\bar{\Theta}, \mathbb{R}, 0)$. For $g \in C_{0}^{\alpha / 2, \alpha}(\bar{\Theta}, \mathbb{R})$ we denote by $V g$ a solution of the problem

$$
\begin{gathered}
\mathcal{L} z(t, x)=g(t, x) \text { in } \Theta, \\
z(t, x)=0 \text { on } \Sigma .
\end{gathered}
$$

In view of classical theory (see [11]) $V g \in C^{1+\alpha / 2,2+\alpha}(\bar{\Theta}, \mathbb{R}, 0)$ is well defined and

$$
\|V g\|_{2+\alpha}^{\bar{\Theta}} \leq c\|g\|_{\alpha}^{\bar{\Theta}}
$$

for some $c \geq 0$, which implies that $V$ is continuous. Now we will construct a bounded linear extension of $V$ into the space $L^{q}(\bar{\Theta}, \mathbb{R})$, for some $q>1$. Since $C_{0}^{\alpha / 2, \alpha}(\bar{\Theta}, \mathbb{R})$ is dense in $L^{q}(\bar{\Theta}, \mathbb{R})$ there exists a sequence $\left\{g_{i}\right\}_{i=1}^{\infty} \subset$ $C_{0}^{\alpha / 2, \alpha}(\Theta, \mathbb{R})$ such that $\left\|g_{i}-g\right\|_{L^{q}(\bar{\Theta}, \mathbb{R})} \rightarrow 0$ as $i \rightarrow \infty$.

By the application of Theorem 5.2 [11] the linear problem (22),(23) (for $\left.g=g_{i}\right)$ has the unique solution $V g_{i} \in C^{1+\alpha / 2,2+\alpha}(\bar{\Theta}, \mathbb{R}, 0)$. But, by the definition, classical solutions of $(22),(23)$ are also generalized solutions of (22),(23). Therefore $V g_{i} \in W_{q}^{1,2}(\Theta, \mathbb{R})$ and

$$
\left\|V g_{i}-V g_{j}\right\|_{W_{q}^{1,2}(\bar{\Theta}, \mathbb{R})} \leq c_{1}\left\|g_{i}-g_{j}\right\|_{L^{q}(\bar{\Theta}, \mathbb{R})}
$$

(see [11]) which shows that $\left\{V g_{i}\right\}_{i=1}^{\infty}$ is a Cauchy sequence in $W_{q}^{1,2}(\bar{\Theta}, \mathbb{R})$ since $\left\{g_{i}\right\}_{i=1}^{\infty}$ is a Cauchy sequence in $L^{q}(\bar{\Theta}, \mathbb{R})$. Therefore there exists $u^{*} \in$ 
$W_{q}^{1,2}(\bar{\Theta}, \mathbb{R})$ such that $\left\|u^{*}-V g_{i}\right\|_{W_{q}^{1,2}(\bar{\Theta}, \mathbb{R})} \rightarrow 0$ as $i \rightarrow \infty$. Put $V^{*} g=u^{*}$. It is easy to check that $u^{*}$ is independent of the choice of $\left\{g_{i}\right\}_{i=0}^{\infty}$. Since (see [11])

and

$$
\left\|V^{*} g-V^{*} \bar{g}\right\|_{W_{q}^{1,2}(\bar{\Theta}, \mathbb{R})} \leq c_{1}\|g-\bar{g}\|_{L^{q}(\Theta, \mathbb{R})}
$$

$$
\left\|V g_{i}\right\|_{W_{q}^{1,2}(\bar{\Theta}, \mathbb{R})} \leq c_{1}\left\|g_{i}\right\|_{L^{q}(\bar{\Theta}, \mathbb{R})}
$$

$V^{*}: L^{q}(\bar{\Theta}, R) \rightarrow W_{q}^{1,2}(\bar{\Theta}, \mathbb{R})$ is bounded and continuous.

Put $q=\frac{n+2}{1-\alpha}$. We will show that $u_{\mid \Sigma}^{*}=0$. Indeed, since $W_{q}^{1,2}(\bar{\Theta}, \mathbb{R})$ is imbedded in $C^{(1+\alpha) / 2,1+\alpha}(\Theta, \mathbb{R})$ (see [10]) we have

$$
\left\|u^{*}\right\|_{0}^{\Sigma}=\left\|u^{*}-V g_{i}\right\|_{0}^{\Sigma} \leq\left\|u^{*}-V g_{i}\right\|_{1+\alpha}^{\bar{\Theta}} \leq c_{2}\left\|u^{*}-V g_{i}\right\|_{W_{q}^{1,2}(\bar{\Theta}, \mathbb{R})}
$$

for some $c_{2} \geq 0$.

Now we prove that $u$ is a classical solution of (3), (4) if and only if $u$ is a solution of

$$
z=\left(V^{*} F\right)(z) .
$$

Indeed, let first assume that $u \in C^{1+\alpha / 2,2+\alpha}(E, \mathbb{R}, 0)$ is a classical solution of $(3),(4)$.

Put $\tilde{u}=\left(V^{*} F\right)(u)$. Since, by Lemma 2.1 and $(21) F u \in C_{0}^{\alpha / 2, \alpha}(\bar{\Theta}, \mathbb{R})$, then $\tilde{u}=V F(u)$ and $\tilde{u}$ is a solution of

$$
\mathcal{L} z(t, x)=(F u)(t, x) \quad(t, x) \in \bar{\Theta}, \quad z(t, x)=0 \quad(t, x) \in \Sigma .
$$

But $u$ also satisfies (25). Therefore by the uniqueness (see [11]) $u=\tilde{u}$ and 24 is proved.

Let now u satisfy (24). Since

$$
I: C(\bar{\Theta}, \mathbb{R}) \rightarrow L^{q}(\bar{\Theta}, R) \text { defined by } I z=z \text { for } z \in C(\bar{\Theta}, \mathbb{R})
$$

is continuous, and

$$
\tilde{I}: W_{q}^{1,2}(\bar{\Theta}, \mathbb{R}) \rightarrow C^{(1+\alpha) / 2,1+\alpha}(\bar{\Theta}, \mathbb{R}),
$$

such that $\tilde{I} z=z$ for $z \in W_{q}^{1,2}(\bar{\Theta}, \mathbb{R})$, is also continuous by Lemma 2.1,

$$
V^{*} F: C^{0,1}(E, \mathbb{R}) \rightarrow C_{\bar{\beta}, \beta}^{(1+\alpha) / 2,1+\alpha}(\bar{E}, \mathbb{R}) \quad \text { is continuous. }
$$

Notice that if $u=\left(V^{*} F\right) u$ then $u \in C^{(1+\alpha) / 2,1+\alpha}(\bar{E}, \mathbb{R}, 0)$ and in view of Lemma 2.1 $F u \in C^{\alpha / 2, \alpha}(\bar{\Theta}, \mathbb{R})$. Therefore,

$$
u=V^{*} F u=(V F) u \in C^{1+\alpha / 2,2+\alpha}(E, \mathbb{R}, 0)
$$

and $u$ satisfies $(3),(4)$.

Let $C_{*}^{0,1}(E, \mathbb{R}, 0)=\left\{z \in C_{*}^{0,1}(E, \mathbb{R}): z_{\mid \Gamma}=0\right\}$. Our next claim is that $G=V^{*} F$ is completely continuous operator from $C_{*}^{0,1}(E, \mathbb{R}, 0)$ into itself.

Indeed, it is clear from Lemma 2.1 and the fact that $C_{\bar{\beta}, \beta}^{(1+\alpha) / 2,1+\alpha}(\bar{E}, \mathbb{R})$ is compactly imbedded in $C_{*}^{0,1}(E, \mathbb{R})$. Let

$$
U=\left\{u \in C_{*}^{0,1}(E, \mathbb{R}, 0):\|u\|_{0}^{E}<\tilde{R}+1,\left\|D_{x} u\right\|_{0}^{\Theta}<\tilde{L}+1\right\}
$$


where $\tilde{R}=R(\sigma, M)$ is defined by (15) and $\tilde{L}$ in Lemma 2.2 .

Of course $0 \in U$ and $U$ is bounded, open subset of $C_{*}^{0,1}(E, \mathbb{R}, 0)$. We will show that for every $u \in \partial U, \lambda \in(0,1) \quad u \neq \lambda G u$. Then by Leray-Schauder theorem $G$ has a fixed point which in view of the first part of the proof is the desired conclusion.

Assume that $\lambda G u=u$ for $u \in \partial U, \lambda \in(0,1)$. Then $\lambda\left(V^{*} F\right) u=$ $V^{*}(\lambda)(u)=u$ is a solution of the following problem

$$
\mathcal{L} u(t, x)=\lambda f\left(t, x, u(t, x), u_{(t, x)}, D_{x} u(t, x)\right) \quad \text { in } \Theta \quad u(t, x)=0 \quad \text { in } \Gamma .
$$

Applying Lemma 2.2 (with $\lambda F, 0$ instead of $F$ and $\Psi$ ) we obtain that $\|u\|_{0}^{E} \leq \tilde{R}$ and $\left\|D_{x} u\right\|_{0}^{\Theta} \leq \tilde{L}$ as $|\lambda|<1$. This contradicts the fact that $u \in \partial U$.

Remark 2.3. If $f$ is Lipschitz continuous in $u$, $w$ (i.e if we put $\alpha=\gamma=1$ only in $\left.A_{\bar{\beta}, \beta} 3\right)$ ) then problem (3),(4) has a unique solution.

The uniqueness of solutions for (3) (4) follows from Remark 1.1, Lemma 2.2 and from Theorem 4 in [15].

\section{IBVP With A DEVIATED ARGUMENT.}

In this paragraph we will look more closely at Example 1.1. Recall some notation.

Let $g: \Theta \times \mathbb{R} \times \mathbb{R} \times \mathbb{R}^{n} \rightarrow \mathbb{R}, \mu: \Theta \rightarrow \mathbb{R}, \nu: \Theta \rightarrow \mathbb{R}^{n}$ and $\Psi$ are given function. Suppose that condition (5) is satisfied. In this section we will consider problem $(6),(7)$.

We will say that IBVP $(6),(7)$ satisfies the compatibility condition if

$$
\begin{gathered}
D_{t} \Psi(0, x)-\sum_{i, j=1}^{n} a_{i j}(0, x) D_{x_{i} x_{j}} \Psi(0, x)-\sum_{i}^{n} b_{i}(0, x) D_{x_{i}} \Psi(0, x) \\
=g\left(0, x, \Psi(0, x), \Psi(\mu(0, x), \nu(0, x)), D_{x} \Psi(0, x)\right)
\end{gathered}
$$

for $x \in \partial \Omega$.

Assumption 3.1. Let $\|\Psi\|_{\Gamma} \leq M, \alpha \in(0,1)$ and $\gamma \geq \alpha$ given constant. Suppose that,

1) there exists $\sigma \in O_{M}$ such that

$$
g(t, x, u, r, 0) \operatorname{sgn}(u) \leq \sigma(t, \max (|u|,|r|)) \quad \text { in } \quad \Theta \times \mathbb{R} \times \mathbb{R}
$$

2) there exists nondecreasing function $\rho: \mathbb{R}_{+} \rightarrow \mathbb{R}_{+}$such that $|g(t, x, u, r, p)| \leq \rho(\max (|u|,|r|))\left(1+|p|^{2}\right) \quad$ in $\quad \Theta \times \mathbb{R} \times \mathbb{R} \times \mathbb{R}^{n} ;$

3) for every $R, L \geq 0$ there exists $\tilde{C}_{R, L} \geq 0$ such that

$$
\begin{aligned}
|f(t, x, u, r, p)-f(\bar{t}, \bar{x}, \bar{u}, \bar{r}, \bar{p})| \leq & \tilde{C}_{R, L}\left(|t-\bar{t}|^{\alpha / 2}+|x-\bar{x}|^{\alpha}+|u-\bar{u}|^{\alpha}\right. \\
& \left.+|r-\bar{r}|^{\gamma}+|p-\bar{p}|\right)
\end{aligned}
$$

in $\Theta \times[-R, R] \times[-R, R] \times B(L)$.

4) there exist $\frac{\alpha}{\gamma(\alpha+1)} \leq \eta_{\mu} \leq 1, \frac{\alpha}{2 \gamma} \leq \eta_{\nu} \leq \frac{1}{2}$ and $H_{\mu}, H_{\nu} \geq 0$ such that 


$$
\begin{aligned}
& |\mu(t, x)-\mu(\bar{t}, \bar{x})| \leq H_{\mu}\left(|t-\bar{t}|^{\eta_{\mu}}+|x-\bar{x}|^{2 \eta_{\mu}}\right) \\
& |\nu(t, x)-\nu(\bar{t}, \bar{x})| \leq H_{\nu}\left(|t-\bar{t}|^{\eta_{\nu}}+|x-\bar{x}|^{2 \eta_{\nu}}\right)
\end{aligned}
$$

on $\Theta$ (if $2 \eta_{\mu}>1$ then $\mu$ does not depend on $x$ ).

5) there exists $\tilde{\Psi} \in C_{\bar{\beta}, \beta}^{1+\alpha / 2,2+\alpha}(E, \mathbb{R})$ such that $\tilde{\Psi}_{\mid \Gamma}=\Psi$ for $\bar{\beta}=\frac{\alpha}{2 \gamma \eta_{\mu}}, \beta=$ $\frac{\alpha}{2 \gamma \eta_{\nu}}$.

Theorem 3.1. Assume that

1) $a_{i j}, b_{i} \in C^{\alpha / 2, \alpha}(\bar{\Theta}, \mathbb{R})$;

2) $\partial \Omega$ belongs to class $C^{2+\alpha}$;

3) $g, \Psi$ satisfy Assumption 3.1 and the problem (6) (7) satisfies the compatibility condition (26).

Then IBVP (6),(7) has a solution $u \in C_{\bar{\beta}, \beta}^{1+\alpha / 2,2+\alpha}(E, \mathbb{R})$. This solution is unique if $g$ is Lipshitz continuous in $u, r$ (locally in $r, u, p$ ).

Proof. Put

$$
f(t, x, u, w, p)=g(t, x, u, w(\mu(t, x)-t, \nu(t, x)-x), p)
$$

for $(t, x, u, w, p) \in \Theta \times \mathbb{R} \times C(D, \mathbb{R}) \times \mathbb{R}^{n}$ It is easy to verify that if $u \in C_{\bar{\beta}, \beta}^{1+\alpha / 2,2+\alpha}(E, \mathbb{R}) \cap C L S(f, \Psi, \mathcal{L})$, then $u$ satisfies $(6),(7)$. In view of Theorem 2.1 it suffices to show that $\mathrm{f}$ satisfies its hypothesis. We will show only $\left.A_{\bar{\beta}, \beta} 4\right)$, which is the most complicated. Let $w \in C^{\bar{\beta}, \beta}(D, \mathbb{R}, q),|p| \leq L$

$$
\begin{gathered}
|f(t, x, u, w, p)-f(\bar{t}, x, u, w, p)| \\
\leq g(t, x, u, w(\mu(t, x)-t, \nu(t, x)-x), p)-g(\bar{t}, x, u, w(\mu(\bar{t}, x)-\bar{t}, \nu(\bar{t}, x)-x), p) \\
\leq \tilde{C}_{q, L}\left(|t-\bar{t}|^{\alpha / 2}+|w(\mu(t, x)-t, \nu(t, x)-x)-w(\mu(\bar{t}, x)-\bar{t}, \nu(\bar{t}, x)-x)|^{\gamma}\right) \\
\leq \tilde{C}_{q, L}\left(|t-\bar{t}|^{\alpha / 2}+\left[H_{t, D}^{\bar{\beta}}(w)\right]^{\gamma}\left[|\mu(t, x)-\mu(\bar{t}, x)|^{\gamma \bar{\beta}}+|t-\bar{t}|^{\gamma \bar{\beta}}\right]\right. \\
\left.+\left[H_{x, D}^{\beta}(w)\right]^{\gamma}|\nu(t, x)-\nu(\bar{t}, x)|^{\gamma \beta}\right) \leq \tilde{C}_{q, L}\left(|t-\bar{t}|^{\alpha / 2}+\left[H_{t, D}^{\bar{\beta}}(w)\right]^{\gamma}\left[H_{\mu}\right]^{\gamma \bar{\beta}}|t-\bar{t}|^{\gamma \bar{\beta} \eta_{\mu}}\right. \\
\left.+\left[H_{t, D}^{\bar{\beta}}(w)\right]^{\gamma}|t-\bar{t}|^{\gamma \bar{\beta}}+\left[H_{x, D}^{\beta}(w)\right]^{\gamma}\left[H_{\nu}\right]^{\gamma \beta}|t-\bar{t}|^{\gamma \beta \eta_{\nu}}\right)
\end{gathered}
$$

Since $\gamma \beta, \gamma \bar{\beta} \geq \alpha / 2$ and $\gamma \bar{\beta} \eta_{\mu}, \gamma \beta \eta_{\nu}=\alpha / 2$ we see that there exist $\tilde{H}_{q, L} \geq 0$ such that

$$
|f(t, x, u, w, p)-f(\bar{t}, x, u, w, p)| \leq \tilde{H}_{q, L}|t-\bar{t}|^{\alpha / 2}
$$

Similary we can show that

$$
|f(t, x, u, w, p)-f(t, \bar{x}, u, w, p)| \leq \hat{H}_{q, L}|x-\bar{x}|^{\alpha}
$$

for some $\hat{H}_{q, L} \geq 0$. This proves $\left.A_{\bar{\beta}, \beta} 4\right)$.

For the uniqueness see Remark 2.3.

Corollary 3.1. (i) If $\gamma=1, \eta_{\mu}=\frac{\alpha}{\alpha+1}, \eta_{\nu}=\frac{\alpha}{2}$ then $\bar{\beta}=\frac{\alpha+1}{2}, \beta=1$. (The function $\Psi$ needs to satisfy Lipschitz condition in $x$ )

(ii) If $\gamma=1 \eta_{\mu}=1(\mu(t, x)=\mu(t)) \eta_{\nu}=\frac{1}{2}$ then $\bar{\beta}=\frac{\beta}{2}=\frac{\alpha}{2}$ 
Remark 3.1. It is also possible, using a similar argument, to obtain a theorem on the existence and uniqueness of classical solutions for the problem (8), (9).

See explanation given to Assumption 2.1.

\section{REFERENCES}

[1] H. Amann, Existence and multiplicity theorems for semi-linear elliptic boundary value problems, Math. Z. 150 (1976), 281-295.

[2] A. Belleni-Morante, An integro-differential equation arising from the theory of heat conduction in rigid materials with memory, Boll. Un. Mat. Ital. 5, 15-B, (1978), 470482.

[3] P. Brandi and R. Ceppitelli, On the existance of solutions of a nonlinear functional partial differential equations of the first order, Atti Sem. Mat. Fis. Univ. Modena, 29 (1980), 166-186.

[4] P. Brandi and R.Ceppitelli, Existence, uniqueness and continuous dependence for a first order nonlinear partial differential equation in a hereditary structure, Ann. Polon. Math. 47 (1986), 121-136.

[5] S. Brzychczy, Chaplygin's method for a system of nonlinear parabolic differentialfunctional equations, Differ. Uravn. 22 (1986), 705-708 (in Russian).

[6] S. Brzychczy, Existence of solutions for non-linear systems of differential-functional equations of parabolic type in an arbitrary domain, Ann. Polon. Math. 47 (1987), 309-317.

[7] S. A. Gourley and N.F.Britton, On a modified Volterra population equation with diffusion, Nonlinear Anal. 21 (1993), 389-395.

[8] J. Hale, Theory of Functional Differential Equations, Springer-Verlag, New York, 1977.

[9] J. K Hale and S. M. V. Lunel, Introduction to Functional Differential Equations, Springer-Verlag, New York, 1993.

[10] G. S. Ladde, V. Lakshmikantham and A. S. Vatsala, Monotone Iterative Techniques for Nonlinear Differential Equations, Pitman Advanced Publishing Program, Boston, 1985 .

[11] 0. A. Ladyzhenskaya, V. A. Solonikov and N. N. Uralceva, Linear and Quasilinear Equation of Parabolic Type, Nauka, Moscow, 1967 (in Russian). (Translation of Mathematical Monographs, 23, Amer. Math. Soc., Providence, 1968.)

[12] C. V. Pao, Coupled nonlinear parabolic systems with time delays, J. Math. Anal. Appl. 196 (1995), 237-265.

[13] J. Szarski, Sur un système non linéaire d'inéqualités différentialles paraboliques contenant des functionnelles, Colloq. Math. 16 (1967), 141-145.

[14] J. Szarski, Uniqueness of solutions of mixed problem for parabolic differentialfunctional equations, Ann. Polon. Math. 28 (1973), 52-65.

[15] K. Topolski, Parabolic differential-functional inequalities in a viscosity sense, Ann. Polon. Math. 68 (1998), 17-25.

[16] H. Wilhelmsson, Solutions of reaction-diffusion integral equations describing explosive evolution of densities for localized structured, J. Math. Phys. 29 (1988), 1776-1779.

INSTITUT OF MATHEMATICS

UNIVERSITY OF GDAŃSK

Wit Stwosz 57

80-952 GDAǸsk, POLAND

E-mail address: matkt@ksinet.univ.gda.pl 


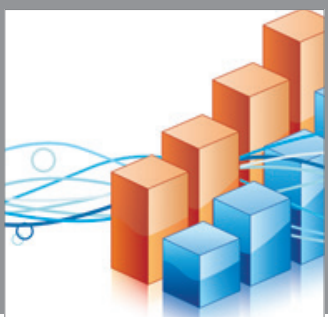

Advances in

Operations Research

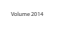

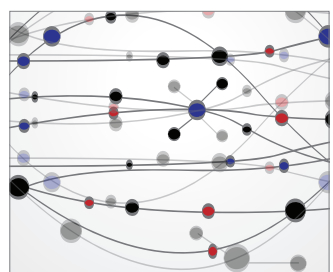

\section{The Scientific} World Journal
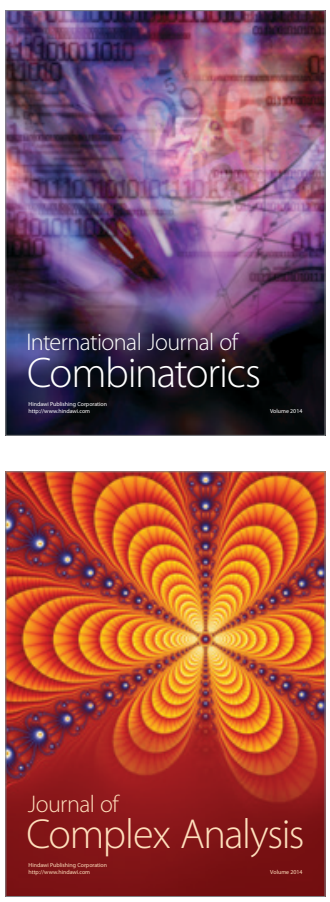

International Journal of

Mathematics and

Mathematical

Sciences
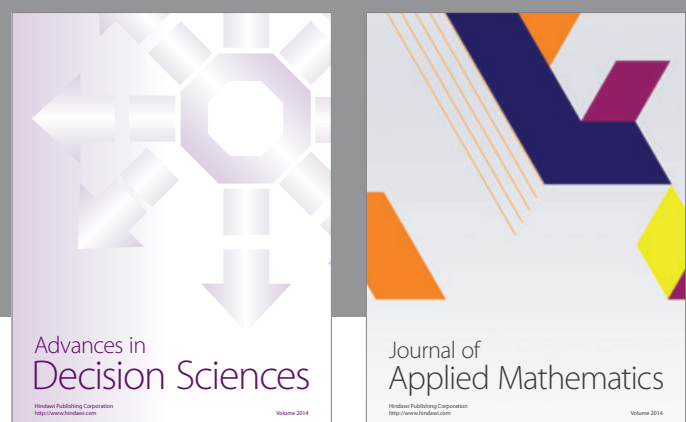

Journal of

Applied Mathematics
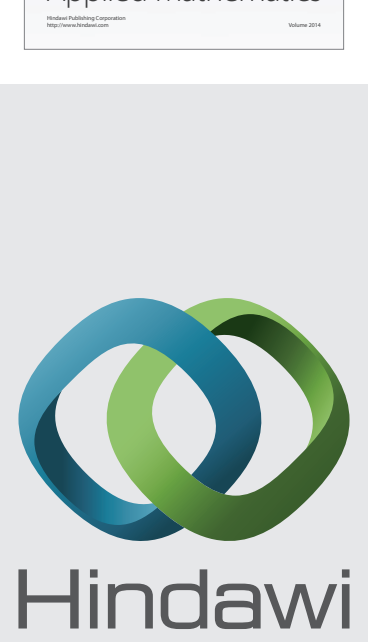

Submit your manuscripts at http://www.hindawi.com
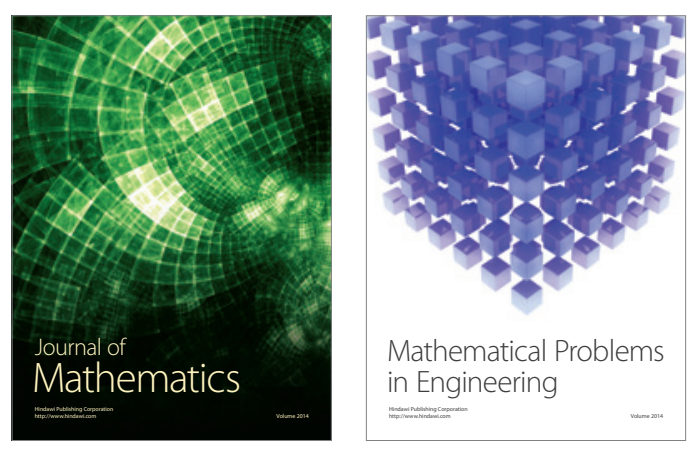

Mathematical Problems in Engineering
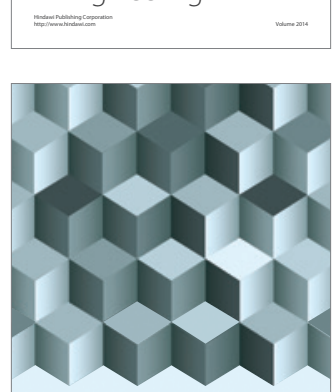

Journal of

Function Spaces
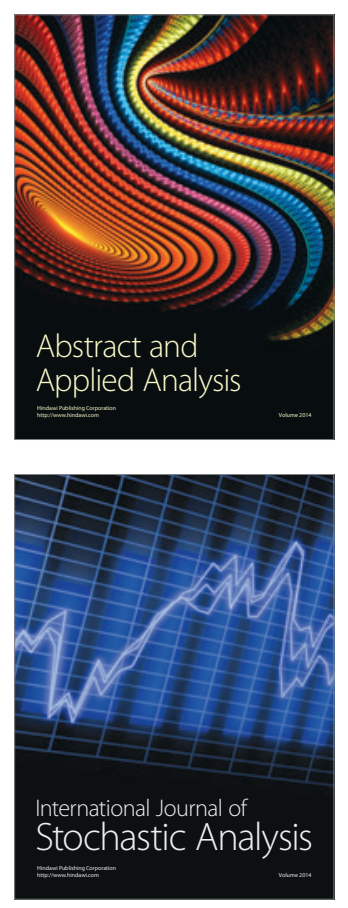

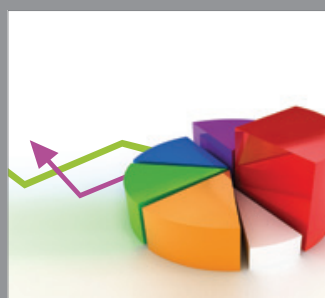

ournal of

Probability and Statistics

Promensencen
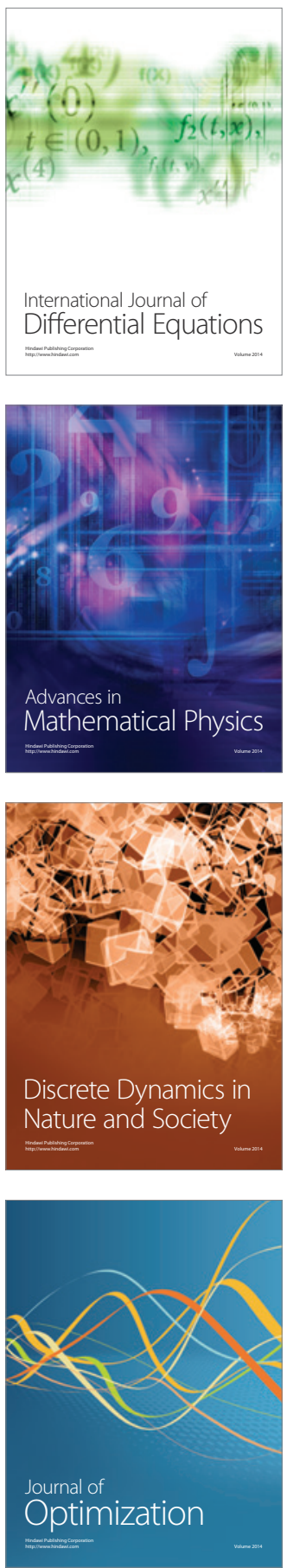